\title{
Present and Future Gamma-Ray Probes of the Cygnus OB2 Environment
}

\author{
Luis A. Anchordoqui, ${ }^{1,2}$ Haim Goldberg, ${ }^{3}$ Russell D. Moore, ${ }^{2}$ \\ Sergio Palomares-Ruiz, ${ }^{4}$ Diego F. Torres, ${ }^{1,5}$ and Thomas J. Weiler ${ }^{6}$ \\ ${ }^{1}$ Institut de Ciències de l'Espai (IEEC-CSIC), Campus UAB, Torre C5, 2a planta, 08193 Barcelona, Spain \\ ${ }^{2}$ Department of Physics, University of Wisconsin-Milwaukee, P.O. Box 413, Milwaukee, WI 53201, USA \\ ${ }^{3}$ Department of Physics, Northeastern University, Boston, MA 02115, USA \\ ${ }^{4}$ Centro de Física Teórica de Partículas, Instituto Superior Técnico, 1049-001 Lisboa, Portugal \\ ${ }^{5}$ Institució Catalana de Recerca i Estudis Avançats (ICREA), Spain \\ ${ }^{6}$ Department of Physics and Astronomy, Vanderbilt University, Nashville, TN 37235, USA
}

(Dated: November 12, 2018)

\begin{abstract}
The MAGIC Collaboration has provided new observational data pertaining to the TeV J2032+4130 gamma-ray source (within the Cygnus OB2 region), for energies $E_{\gamma}>400 \mathrm{GeV}$. It is then appropriate to update the impact of these data on gamma-ray production mechanisms in stellar associations. We consider two mechanisms of gamma-ray emission, pion production and decay (PION) and photoexcitation of high-energy nuclei followed by prompt photo-emission from the daughter nuclei $\left(A^{\star}\right)$. We find that while the data can be accommodated with either scenario, the $A^{\star}$ features a spectral bump, corresponding to the threshold for exciting the Giant Dipole Resonance, which can serve to discriminate between them. We comment on neutrino emission and detection from the region if the PION and/or $A^{\star}$ processes are operative. We also touch on the implications for this analysis of future Fermi and Čerenkov Telescope Array data.
\end{abstract}

PACS numbers: 07.85.-m, 98.70.Sa

CFTP/09-27

Two well-known mechanisms for generating $\mathrm{TeV} \gamma$-rays in astrophysical sources are the purely electromagnetic (EM) one proceeding via synchrotron emission and inverse Compton scattering, and the hadronic (PION) one in which $\gamma$-rays originate from $\pi^{0}$ production and decay [1, 2]. Recently, we highlighted a third dynamic which leads to $\mathrm{TeV} \gamma$-rays: photo-excitation of highenergy nuclei, followed by prompt photo-emission from the excited daughter nuclei [3, 44]. In this chain reaction, the nuclei act in analogy to Einstein's relativistic moving mirror to "double-boost" eV starlight to TeV energies for a Lorentz boost factor $\gtrsim 10^{6}$. The important role played by the Giant Dipole Resonance (GDR) in the photo-disintegration effectively suppresses the contribution to the $\gamma$-ray spectrum below a TeV [5]. This process (which we have denoted by $A^{\star}$ ) has been proposed [6] as a candidate explanation of the unidentified HEGRA source TeV J2032+4130 [7] at the edge of the Cygnus OB2 (Cyg OB2) association (see Ref. [8] for the discussion regarding the HESS source HESS J1023-575 at the edge of Westerlund 2 [9]). This stellar association has been known to harbor a large population of massive and early type stars [10] that can provide the required UV target density.

Recently, the MAGIC Collaboration has reported new $\mathrm{TeV} \gamma$-ray data from this region [11]. Thus, it is of interest to expand previous analyses to include this new data. In this paper, we present a unified analysis of the $A^{\star}$ and PION mechanisms for gamma-ray production in Cyg OB2. This combined analysis indicates the relative importance of these two mechanisms. Extrapolation to lower energies then allows us to make predictions within reach of the Fermi mission [12].

$\mathrm{TeV}$ gamma-ray data from this region has also been reported by the Milagro Collaboration [13]. However, inclusion of these data in our study will require further analysis to distinguish contributions from the HEGRA source and diffuse interactions expected from the larger region of observation. Thus we postpone the consideration of the Milagro data.

The critical parameters for the PION and $A^{\star}$ mechanisms are the ambient hydrogen gas density and the UV photon background, respectively. These parameters at present are subject to considerable uncertainty. It is a further goal of this work to see whether the present and evolving gamma-ray data can meaningfully constrain these parameters.

The stellar distribution of Cyg OB2 reveals a rather regular and almost circular density profile with the center located at $(\alpha, \delta)=\left(20^{h} 33^{m} 10^{s},+41^{\circ} 12^{\prime}\right)$ and with a pronounced maximum slightly offset at $(\alpha, \delta)=$ $\left(20^{h} 33^{m} 10^{s},+41^{\circ} 15.7^{\prime}\right)[10$. Star counts show that $50 \%$ of the members are located within a radius of $21^{\prime}$, and $90 \%$ within a radius of $45^{\prime}$ around the center. By integrating the radial density profile, after subtraction of the field star density, the total number of OB stars is found to be $2600 \pm 400$, with a O-type star population of $120 \pm 20$. This suggests that the total mass of the association is about $10^{4} M_{\odot}$. Distance determinations set the proximity of the Cyg OB2 to $d \sim 1.7 \mathrm{kpc}[14$. At such distance, the inner $21^{\prime}$, with half the total number of objects, results in a physical radius of $R_{\text {in }} \sim 10 \mathrm{pc}$, with $R_{\text {out }} \sim 30$ pc being the radius of the association. Projected onto the sky at the distance of Cyg OB2, the HEGRA/MAGIC signal from TeV J2032+4130 [7, 11] was observed only in a $3 \mathrm{pc}$ radius cell at the edge of the association. With the same angular radius, there are a total of $\sim 14$ cells in the core of the association. The flux 
in each of these cells is bounded $\sim 1 \%$ of Crab, about 3 times less than that of the TeV J2032+4130 cell. On the other hand, the age of the stellar association is supposed to be 2-4 Myr 15.

As mentioned above, the prediction of the PION $\gamma$-ray yield is subject to uncertainty in the ambient gas density (as well as the ambient cosmic ray flux). We will find that for gas densities larger than $0.1 \mathrm{~cm}^{-3}$ in the vicinity of the source, the PION mechanism will dominate, with the $A^{\star}$ mechanism assuming dominance for smaller densities. A considerably higher density, $n_{\mathrm{H}}=30 \mathrm{~cm}^{-3}$, has been estimated from observations of the $\mathrm{CO} J=1 \rightarrow 0$ rotational transition [19]. If taken as representing an average over the core of the association, this value implies a hydrogen gas mass of $3000 M_{\odot}$, which is about $30 \%$ of the mass found in stars. Arguments have been given [20] that this estimated density [19] should be interpreted as an upper bound. Among concerns are the $\mathrm{CO}-\mathrm{H}_{2}$ conversion factor [21], and the size of the region used for averaging. More recently, a thorough analysis of the region, with higher angular resolution observations of ${ }^{12} \mathrm{CO}$ and ${ }^{13} \mathrm{CO}$ has been presented [22]. Of interest in these results is a significant ${ }^{13} \mathrm{CO}$ cavity right at the $\mathrm{TeV}$ source position. This is reminiscent of a formerly found IR void. Only one ${ }^{13} \mathrm{CO}$ clump is seen at a position consistent with the projected position of HEGRA source. No claim is made as to any physical connection between the clump and the cavity. Although the clump is massive (claimed mass at $337 \mathrm{M}_{\odot}$ ), its size is significantly smaller than the size of the extended $\mathrm{TeV}$ source. If gamma-rays were to originate from interactions with just this clump, there is no reason for the source to appear extended in instruments such as MAGIC [23] or VERITAS 24].

We adopt as our fiducial density the low estimate $n_{\mathrm{H}} \sim$ $0.1 \mathrm{~cm}^{-3}$, which agrees with the analysis in Ref. [20]. This choice allows us to illustrate the crossover point of PION dominance versus $A^{\star}$ dominance. It is conservative and wise at this point to depend on future experiments (discussed below) to resolve the issue of PION versus $A^{\star}$ dominance.

First we discuss the PION mechanism for $\mathrm{TeV}$ gamma-ray production. The emissivity (number/volume/time/energy) of neutral pions resulting from an isotropic distribution of highly relativistic nuclei having a power-law energy spectrum $d n_{A}\left(E_{N}\right) / d E_{N}=N_{A}\left(E_{N} / E_{0}\right)^{-\alpha}$, colliding with ambient hydrogen, is given by [1]

$$
\begin{aligned}
Q_{\pi^{0}}^{A p}\left(E_{\pi^{0}}\right) & =c n_{\mathrm{H}} \int_{E_{N}^{\mathrm{th}}\left(E_{\pi^{0}}\right)}^{E_{N}^{\max }} \frac{d n_{A}}{d E_{N}}\left(E_{N}\right) \\
& \times \frac{d \sigma_{A}}{d E_{\pi^{0}}}\left(E_{\pi^{0}}, E_{N}\right) d E_{N}
\end{aligned}
$$

where $N_{A}$ is the normalization constant with units $1 /$ volume/energy, $E_{0}$ is set to $1 \mathrm{TeV}, E_{N}^{\mathrm{th}}\left(E_{\pi^{0}}\right)$ is the minimum energy per nucleon required to produced a pion with energy $E_{\pi^{0}}$, and $d \sigma_{A}\left(E_{\pi^{0}}, E_{N}\right) / d E_{\pi^{0}}$ is the differential cross section for the production of a pion with en- ergy $E_{\pi^{0}}$ in the lab frame due a nucleus $A$ of energy per nucleon $E_{N}=E_{A} / A$ colliding with a hydrogen atom at rest. The differential cross-section can be parametrized by

$$
\frac{d \sigma_{A}}{d E_{\pi^{0}}}\left(E_{\pi^{0}}, E_{N}\right) \simeq \frac{\sigma_{0}^{A}}{E_{\pi^{0}}} x F_{\pi^{0}}\left(x, E_{N}\right),
$$

where $x \equiv E_{\pi^{0}} / E_{N}, \sigma_{0}^{A}=A^{3 / 4} \sigma_{0}$ provides a scaling of the cross-section with the atomic number 25], $\sigma_{0}=$ $\left(34.3+1.88 L+0.25 L^{2}\right) \mathrm{mb}$, and $F_{\pi^{0}}\left(x, E_{N}\right) \equiv d N_{\pi^{0}} / d x$ is a fragmentation function. We take

$$
\begin{aligned}
F_{\pi^{0}}\left(x, E_{N}\right) & =4 \beta B_{\pi} x^{\beta-1}\left(\frac{1-x^{\beta}}{1+r x^{\beta}\left(1-x^{\beta}\right)}\right)^{4} \\
& \times\left(\frac{1}{1-x^{\beta}}+\frac{r\left(1-2 x^{\beta}\right)}{1+r x^{\beta}\left(1-x^{\beta}\right)}\right),
\end{aligned}
$$

with $B_{\pi}=a+0.25, \beta=0.98 / \sqrt{a}, r=2.6 / \sqrt{a}, a=3.67+$ $0.83 L+0.075 L^{2}$, and $L=\ln \left(E_{N} / \mathrm{TeV}\right)$ 26]. Because isotropy is implied in (11), it is straightforward to obtain the $\gamma$-ray emissivity [6]; it is

$$
Q_{\gamma}^{A p}\left(E_{\gamma}\right)=2 \int_{E_{\pi^{0}}^{\min }\left(E_{\gamma}\right)}^{E_{\pi^{0}}^{\max }\left(E_{N}^{\max }\right)} \frac{Q_{\pi^{0}}^{A p}\left(E_{\pi^{0}}\right)}{\left(E_{\pi^{0}}^{2}-m_{\pi}^{2}\right)^{1 / 2}} d E_{\pi^{0}}
$$

where $E_{\pi^{0}}^{\min }\left(E_{\gamma}\right)=E_{\gamma}+m_{\pi}^{2} /\left(4 E_{\gamma}\right)$.

Before proceeding to the $A^{\star}$ mechanism, we pause to compare Eq. (3), which is a functional fit to the outcome of numerical simulations obtained with the SIBYLL event generator 27], to data collected at Tevatron by the CDF detector 28]. The results of simulations leading to Eq. (3) have been reported at nucleon energies of $0.1 \mathrm{TeV}$ and $1000 \mathrm{TeV}$. The latter corresponds to a center-of-mass energy of $\sqrt{s} \simeq 1410 \mathrm{GeV}$. The CDF group at Tevatron has measured the charged pion spectrum for pseudorapidity $|\eta|<3.5$, at $\mathrm{cm}$ energies of $630 \mathrm{GeV}$ and $1800 \mathrm{GeV}$. They have provided a fit over the energy range of interest, quadratic in $\ln \left[s / \mathrm{GeV}^{2}\right]$, with $\chi^{2}=0.72$ for three degrees of freedom

$$
\begin{aligned}
\frac{d N_{\mathrm{ch}}}{d \eta} & =(0.023 \pm 0.008) \ln ^{2} s-(0.25 \pm 0.19) \ln s \\
& +(2.5 \pm 1.0)
\end{aligned}
$$

valid for $\eta=0$. Taking into account that $F_{\pi^{+}} \simeq F_{\pi^{-}} \simeq$ $F_{\pi^{0}}$, that the spectral dependence on $\eta$ is mild, and that

$$
\frac{d N_{\pi^{0}}}{d \eta} \simeq x \frac{d N_{\pi^{0}}}{d x}=x F_{\pi^{0}}
$$

we find that the results in Eq. (3) agree remarkably, within one standard deviation, to the CDF fit to their Tevatron data.

Now we discuss the $A^{\star}$ mechanism of $\mathrm{TeV}$ gammaray production. The photo-excitation (or photodisintegration) rate for a highly relativistic nucleus with 
energy $E=\gamma A m_{N}$ (where $\gamma$ is the Lorentz factor) propagating through an isotropic photon background with energy $\epsilon$ and number-density spectrum $n(\epsilon)$ is 29]

$$
R_{A}=\frac{1}{2} \int_{0}^{\infty} \frac{n(\epsilon)}{\gamma^{2} \epsilon^{2}} d \epsilon \int_{0}^{2 \gamma \epsilon} \epsilon^{\prime} \sigma_{A}\left(\epsilon^{\prime}\right) d \epsilon^{\prime},
$$

where $\sigma_{A}\left(\epsilon^{\prime}\right)$ is the cross section for photo-disintegration of a nucleus of mass $A$ by a photon of energy $\epsilon^{\prime}$ in the rest frame of the nucleus.

We assume that $n(\epsilon)$ results from thermal emission of the stars in the whole Cyg OB2 association, out to $R_{\text {out }} \sim 30$ pc. We model the association with half of the stars uniformly distributed in the inner region, $R_{\mathrm{in}} \sim 10 \mathrm{pc}$, and the other half uniformly distributed in the outer shell, i.e. the density of stars in the inner region is $\left(R_{\text {out }} / R_{\text {in }}\right)^{3}-1 \sim 26$ times that in the outer shell. To reproduce the size and position of the source of the HEGRA signal, the photo-disintegration must occur in a region of radius $r \sim 3 \mathrm{pc}$ at the edge of the inner part of the association, $R \leq R_{\text {in }}$. The average photon density in this region reflects both the temperatures $T_{\mathrm{O}}$ and $T_{\mathrm{B}}$ of the $\mathrm{O}$ and $\mathrm{B}$ stars, respectively, and dilution resulting from inverse square law considerations. The resulting photon density is

$$
n^{\star}(\epsilon)=\frac{47}{4}\left[\frac{n_{\mathrm{O}}(\epsilon) N_{\mathrm{O}} R_{\mathrm{O}}^{2}+n_{\mathrm{B}}(\epsilon) N_{\mathrm{B}} R_{\mathrm{B}}^{2}}{R_{\text {out }}^{2}}\right],
$$

where $N_{\mathrm{O}(\mathrm{B})}$ is the number of $\mathrm{O}(\mathrm{B})$ stars, $R_{\mathrm{O}(\mathrm{B})}$ is the $\mathrm{O}$ (B) star average radius, and

$$
n_{\mathrm{O}(\mathrm{B})}(\epsilon)=(\epsilon / \pi)^{2}\left[e^{\epsilon / T_{\mathrm{O}(\mathrm{B})}}-1\right]^{-1},
$$

is the Bose-Einstein distribution of photons emitted from a star at temperature $T_{\mathrm{O}(\mathrm{B})}$. The factor $47 / 4$ is a consequence of averaging the inverse square distance within this distribution for the density and the region where the reaction takes place [6]. It is clear, however, that within the 3 pc HEGRA hot spot the concentration of stars would be above average, and thus hereafter we take as a fiducial value for $n^{\mathrm{HEGRA}}(\epsilon)=1.7 n^{\star}(\epsilon)$. The 1.7 factor encapsulates an uncertainty of $\sim 1$ to 2.5 [30]. The resulting photo-disintegration rate $R_{A}$ for the value of this density will be denoted by $R_{A}^{\mathrm{HEGRA}}$.

In Fig. 1 we show the dependence on the Lorentz factor of $R_{A}^{\mathrm{HEGRA}}$, for the stellar ambiance described above. For the O stars we have taken $N_{\mathrm{O}}=130$, a surface temperature $T_{O}=40000 \mathrm{~K}$, and radius $R_{O}=19 R_{\odot}$; for the cooler B stars we assign $T_{B}=18000 \mathrm{~K}, N_{\mathrm{B}}=2470$, and radius $R_{B}=8 R_{\odot}$. The numbers $N_{\mathrm{O}}$ and $N_{\mathrm{B}}$ are consistent with the Cyg OB2 data discussed in the introduction. The cross section has been calculated in both the Narrow-Width Approximation (NWA) and the more accurate dipole approximation, for the nuclear parameters given in Ref. [6]. In the calculation that follows we adopt the more accurate dipole form for the cross section.

The low-energy cutoff on $R_{A}^{\mathrm{HEGRA}}$ is evident in Fig. 1 This cutoff will be mirrored in the resulting gamma-ray

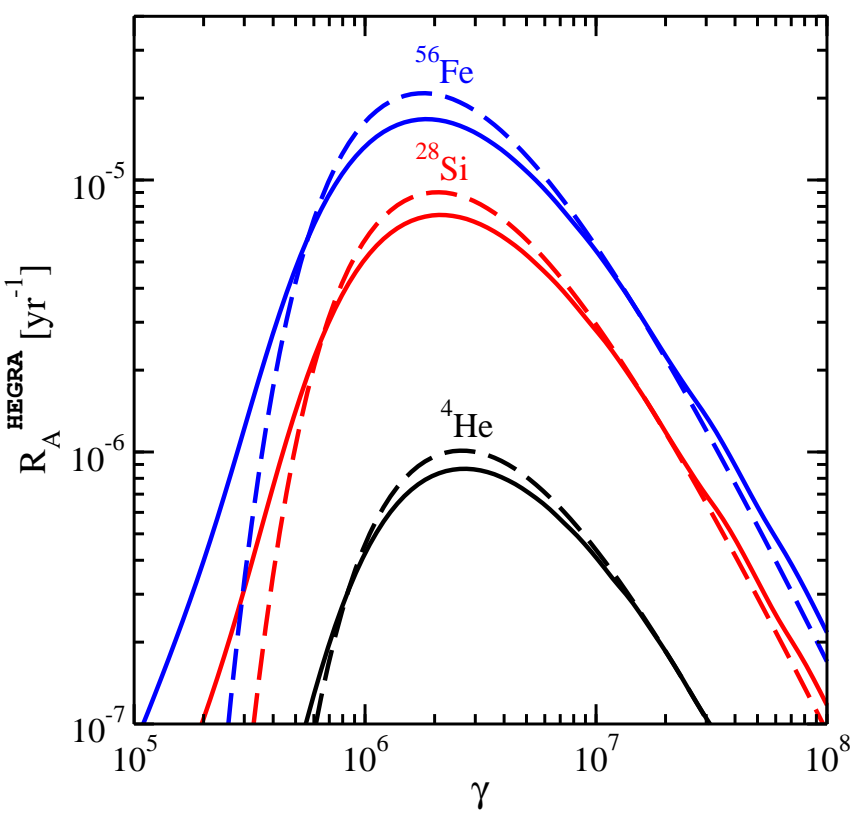

FIG. 1: Photo-disintegration rates of ${ }^{56} \mathrm{Fe},{ }^{28} \mathrm{Si}$, and ${ }^{4} \mathrm{He}$ for the HEGRA hot spot. We have approximated the cross section of the GDR by a dipole (solid-lines) and by a single pole of the NWA (dash-lines).

distribution. Notice that the NWA, which we do not use below, overestimates the severity of the low-energy cutoff.

The energy behavior for photons in the $0.5-10 \mathrm{TeV}$ region of the HEGRA and MAGIC data is a complex convolution of the energy distributions of the various nuclei participating in the photo-disintegration, with the rate factors appropriate to the $\mathrm{eV}$ photon density for the various stellar populations. Approximating the $\gamma$-ray spectrum as being monochromatic with energy $\overline{E_{\gamma A}^{\prime}}$ (in the nucleus rest frame), the emissivity becomes [6]

$$
\begin{aligned}
Q_{\gamma}^{A^{*}}\left(E_{\gamma}\right) & =\sum_{A} \frac{\overline{N_{A}} m_{N}}{2 \overline{E_{\gamma A}^{\prime}}} \int_{\frac{m_{N} E_{\gamma}}{2 \bar{E}_{\gamma A}^{\prime}}} \frac{d E_{N}}{E_{N}} \\
& \times R_{A}^{\mathrm{HEGRA}}\left(E_{N}\right) \frac{d n_{A}}{d E_{N}}\left(E_{N}\right),
\end{aligned}
$$

where $E_{\gamma}$ is the energy of the emitted $\gamma$-ray in the lab, and $\overline{N_{A}}$, which we take to be 2 [31], is the mean $\gamma$-ray multiplicity for a nucleus with atomic number $A$.

It is important to note that the same nucleus source density $d n_{A} / d E_{N}$ is present in the $A^{\star}$ emissivity (10) and in the PION emissivity (4) (via (11)). Thus, a comparison of the two mechanisms will depend only weakly on the exact features of $d n_{A} / d E_{N}$.

The differential photon flux at the observer's site (assuming there is no absorption) receives contributions from both mechanisms, PION and $A^{\star}$. The result is re- 


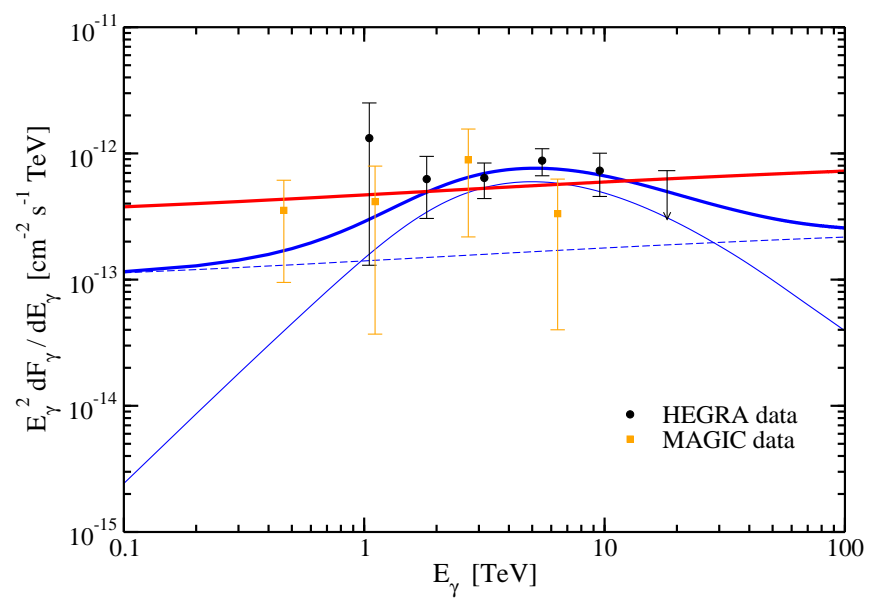

FIG. 2: Eyeball fits to HEGRA and MAGIC $\gamma$-ray spectrum. We have assumed an iron nuclei population with $\alpha=2$ and $\overline{E_{\gamma \mathrm{Fe}}^{\prime}}=2 \mathrm{MeV}$. The thick straight red line is a representative fit to the combined spectra assuming the PION process only with $n_{\mathrm{H}}=2 \mathrm{~cm}^{-3}$ and $N_{\mathrm{Fe}}=5 \times 10^{-12} \mathrm{~cm}^{-3} \mathrm{TeV}^{-1}$. The thick solid blue curve is a similar fit combining both the $A^{*}$ mechanism (thin solid blue line) and PION mechanism (dashed blue line) for $n_{\mathrm{H}}=0.1 \mathrm{~cm}^{-3}$ and $N_{\mathrm{Fe}}=$ $3 \times 10^{-11} \mathrm{~cm}^{-3} \mathrm{TeV}^{-1}$.

lated to to the $\gamma$-ray emissivity as

$$
\frac{d F_{\gamma}}{d E_{\gamma}}\left(E_{\gamma}\right)=\frac{V_{\mathrm{dis}}}{4 \pi d^{2}}\left[Q_{\gamma}^{A p}\left(E_{\gamma}\right)+Q_{\gamma}^{A^{\star}}\left(E_{\gamma}\right)\right]
$$

where $V_{\text {dis }}$ is the volume of the source region and $d$ is the distance to the observer. In Fig. 2 we provide a some eyeball fits (thick solid lines) to the combined HEGRA/MAGIC $\gamma$-ray spectrum, obtained from integrations implicit in the two emissivities in Eq. (11).

The fits are for an iron nuclei population with spectral index $\alpha=2$ and for an average energy of the photon (in the nuclear rest frame) emitted during photoemission $\overline{E_{\gamma \mathrm{Fe}}^{\prime}}=2 \mathrm{MeV}$ [31]. The solid thick blue curve is a fit using both the $A^{*}$ mechanism (solid blue thin line) and PION mechanism (dash blue thin line), with $n_{\mathrm{H}}$ equal to our fiducial value, $0.1 \mathrm{~cm}^{-3}$, and $N_{\mathrm{Fe}}=3 \times 10^{-11} \mathrm{~cm}^{-3} \mathrm{TeV}^{-1}$. The red thick straight line is a representative fit to the combined spectral data assuming the PION process only with $n_{\mathrm{H}}=2 \mathrm{~cm}^{-3}$ and $N_{\mathrm{Fe}}=5 \times 10^{-12} \mathrm{~cm}^{-3} \mathrm{TeV}^{-1}$. For the iron nuclei population assumed in the fits, the target gas density for PION dominance at all energies is $n_{\mathrm{H}} \gtrsim 0.5 \mathrm{~cm}^{-3}$.

Additional data is becoming available from observations of the Fermi satellite. A preliminary measurement in the Cygnus region yields an integrated gamma-ray flux 32.

$$
F_{\gamma}(1-100 \mathrm{GeV}) \simeq 3.07 \times 10^{-8} \mathrm{~cm}^{-2} \mathrm{~s}^{-1} .
$$

If one naïvely assumes a spectrum $\propto E_{\gamma}^{-2}$ for their ob- servations, a squared-energy weighted differential flux of

$$
E_{\gamma}^{2} \frac{d F_{\gamma}}{d E_{\gamma}}=3.07 \times 10^{-11} \mathrm{~cm}^{-2} \mathrm{~s}^{-1} \mathrm{TeV}
$$

is obtained. This is nearly two orders of magnitude above extrapolation of the HEGRA/MAGIC measurement at $100 \mathrm{GeV}$. The presence of low energy powerful sources can clearly dominate the flux at lower energies [33]. Thus, the normalization inferred from this low-energy data can grossly over-estimate the predicted flux at $100 \mathrm{GeV}$ (see Ref. [34] for a thorough discussion of these issues). A prime candidate source for the low energy radiation is a pulsar (with spin-down power $2.6 \times 10^{35} \mathrm{ergs}^{-1}$ ), which coincides (within errors) with the position of $\mathrm{TeV} \mathrm{J} 2032+4130$ ( $4^{\prime}$ displacement) 35]. The observed $\mathrm{GeV}$ emission can plausibly be ascribed to electron acceleration in the magnetosphere. This radiation is exponentially cut off in the $\mathrm{TeV}$ region. However, such very high energy radiation, which is the focus of the present paper, could possibly be associated with inverse Compton scattering of the electrons which power the pulsar wind nebula, if such exists (see as an example the case of HESS J1825-137 [36], and many others in the recent literature). Alternatively, the $\mathrm{TeV}$ radiation can originate in the OB association via the $A^{\star}$ and PION mechanisms.

We now conclude with a discussion of our results:

- From Fig. 2 it is apparent that the combined HEGRA/MAGIC data can be fit with only the PION mechanism in operation. Such a fit applies if the gas density $n_{H}$ is larger than $2 \mathrm{~cm}^{-3}$. For $0.05 \mathrm{~cm}^{-3} \lesssim n_{H} \lesssim 2 \mathrm{~cm}^{-3}$, a combination of PION and $A^{\star}$ can provide a satisfactory fit to the data, whereas for $n_{\mathrm{H}}<0.05 \mathrm{~cm}^{-3}$ a good fit to all the data can be obtained using only the $A^{\star}$ mechanism.

- The average energy of the photon (in the nucleus rest frame) emitted during photo-emission has been taken as $2 \mathrm{MeV}$. This is appropriate for iron nuclei. If $n_{\mathrm{H}}<0.05 \mathrm{~cm}^{-3}$, a better fit to all the data can be obtained using only the $A^{\star}$ mechanism with a lower average energy of $1.5 \mathrm{MeV}$.

- For low gas densities, the spectral features characteristic of the $A^{\star}$ mechanism become visible. These are best described as a broad bump in the spectrum in the region $1-10 \mathrm{TeV}$.

- In completing the explanation of the HEGRA and MAGIC signal, there remains one issue to address the signal was observed only in a $3 \mathrm{pc}$ radius cell at the edge of the inner association. At this point in our understanding we can provide only qualitative remarks. One possibility is an increased density of very hot OB stars in the TeV J2032+4130 cell, which provide efficient trapping and accelerating conditions for the nuclei, as well as a hot photon 
background. Indeed, a recent estimate [37] indicates around $10 \mathrm{O}$ stars in the region of the source, a number which is a factor of 3 larger than that expected on the basis of a uniform population.

- If the energy spectrum of cosmic electrons $\propto E_{e}^{-2}$ (with an exponential cutoff at $40 \mathrm{TeV}$ ), the data can also be explained by inverse Compton scattering of these electrons on the cosmic microwave background photons [1]. The EM explanation can only accommodate the data if the Compton peak is matched to the energy range of HEGRA/MAGIC detection, a possibility allowed within errors.

- We expect a flux of $\mathrm{TeV} \nu_{\mu}, \bar{\nu}_{\mu}$ from both the $A^{\star}$ (via neutron decay followed by oscillations [38]) and PION (via $\pi^{ \pm}$decay) [20] mechanisms. Allowing about one muon neutrino per photon after oscillation, we expect about 1.2 events/yr at IceCube with a background from atmospheric neutrinos of about 1 event/yr [39]. However, it is possible that this event rate can be considerably enhanced by emission from the additional 3-pc cells in the association (which will not be resolved by future neutrino detectors). The signal enhancement can amount to as much as a factor of about 5 due to the emission at the upper limit value set by gamma-ray observation from each cell in the rest of region (e.g., MILAGRO measurement in a region centered in the HEGRA region but ten times larger [13] and MAGIC upper limit in the direction of Cyg X3 [11], which approximately coincides with that of Cyg OB2). Such accumulation could make the source visible in neutrinos at IceCube. We also note that absorption of gamma-rays at the center of the association (see e.g., Ref. [40]) could be relevant, implying an even higher neutrino flux from some cells. Observation of a neutrino flux from the HEGRA/MAGIC source could disqualify an EM explanation of the origin of the gamma-rays, at least for this source.
- The future Čerenkov Telescope Array [41] will provide stronger spectral discrimination between the $\mathrm{PION}$ and $\mathrm{PION}+A^{\star}$ mechanisms. This telescope is projected to have a factor $>10$ larger sensitivity than MAGIC/VERITAS at TeV energies. It will also cover the lower $\mathrm{GeV}$ energy region (down to tenths of a GEV) where the $A^{\star}$ mechanism is suppressed, thus allowing the possibility of comparing the two mechanisms with a single data set covering the entire energy region of interest. Due to its superb angular resolution (expected perhaps at a factor of 2 or 3 better than that of MAGIC) and field of view (several degrees), it will become the ideal instrument to distinguish emission components in this energy region, and to study morphology of the radiation from $\mathrm{TeV} \mathrm{J} 2032+4130$.

\section{Acknowledgments}

We thank Jordi Isern for a valuable communication. LAA is supported by the US National Science Foundation (NSF) Grant No PHY-0757598, the UWM Research Growth Initiative, and Consejo Superior de Investigaciones Científicas (CSIC). HG is supported by the US NSF Grant No PHY-0757959. SPR is partially supported by the Portuguese FCT through CERN/FP/83503/2008 and CFTP-FCT UNIT 777, which are partially funded through POCTI (FEDER), and by the Spanish Grant FPA2008-02878 of the MCT. DFT is supported by Spanish Grants AYA2006-00530 and AYA2008-01181-E/ESP. TJW was supported by the US Department of Energy (DoE) Grant DE-FG05-85ER40226, an Alexander von Humboldt Foundation Senior Research Award, the faculty leave program of Vanderbilt University, and the hospitality of the Technische Universität Dortmund, and the Max-Planck-Instituts für Physik (Heisenberg-Institut), München, and für Kernphysik, Heidelberg. HG, SPR, and TJW thank the Aspen Center for Physics where this paper was finished.
[1] F. W. Stecker, Cosmic Gamma Rays, Baltimore: Mono Book Co. (1971).

[2] F. A. Aharonian, Very high energy cosmic gamma radiation: A crucial window on the extreme universe, (Singapore: World Scientific Publishing, 2004).

[3] L. A. Anchordoqui, J. F. Beacom, H. Goldberg, S. Palomares-Ruiz and T. J. Weiler, Phys. Rev. Lett. 98, 121101 (2007) arXiv:astro-ph/0611580, S. PalomaresRuiz, J. Phys. Conf. Ser. 60, 195 (2007).

[4] This mechanism was emphasized many years ago by I. V. Moskalenko, [PhD Thesis, Moscow State University, Moscow, 1985], but largely ignored by the rest of the $\gamma$-ray community. See also, V. V. Balashov, V. L. Korotkikh and I. .V. Moskalenko, Moscow University Phy. Bull. 42, 93 (1987); V. V. Balashov, V. L. Korotkikh and I. .V. Moskalenko, Tsirkulyar Astron. Inst. AN
UzbekSSR, Tashkent, No. 124, 471, 3 (1987); V. V. Balashov, Proc. 18th Int. Symp. on Nucl. Phys., Dresden, Germany, ZfK-646, 69, 1988; V. V. Balashov, V. L. Korotkikh and I. .V. Moskalenko, Report at the All Union meeting on gamma-astronomy, Nor-Amberd, USSR, 1988; V. V. Balashov, Proc. 4th Workshop on Perspectives in Nuclear Physics at Intermediate Energies, Trieste, Italy, May 8-12, 1989, p. 503; V. V. Balashov, V. L. Korotkikh and I. .V. Moskalenko, Proc. 21st Int. Cosmic Ray Conf., Adelaide, Australia 2, 416 (1990); S. Karakula, G. Kociolek, I. V. Moskalenko and W. Tkaczyk, Proc. 22nd Int. Cosmic Ray Conf., Dublin, Ireland 1, 536 (1991); S. Karakula, G. Kociolek, I. V. Moskalenko and W. Tkaczyk, Astrophys. J. Suppl. 92, 481 (1994). The mechanism was discussed for gamma-ray bursts, with boost factors of $10^{2}-10^{3}$, 
in N. J. Shaviv and A. Dar, arXiv:astro-ph/9606032 N. J. Shaviv and A. Dar, Proc. of VIIIth Rencontres De Blois, Blois, France, June 6-12, 1996 arXiv:astro-ph/9608135.

[5] A related nuclear model that does not depend on the GDR or the ambient starlight density has been proposed very recently by $\mathrm{K}$. Ioka and $\mathrm{P}$. Meszaros, arXiv:0901.0744 [astro-ph.HE]. This model is based on the observation that some fraction of accelerated nuclei are expected to be naturally excited. In this model, the emitted gamma-ray spectrum would roughly follow the excited nuclear spectrum, even to sub- $\mathrm{TeV}$ energies.

[6] L. A. Anchordoqui, J. F. Beacom, H. Goldberg, S. Palomares-Ruiz and T. J. Weiler, Phys. Rev. D 75, 063001 (2007) arXiv:astro-ph/0611581; H. Goldberg, J. Phys. Conf. Ser. 60, 199 (2007).

[7] F. A. Aharonian et al. [HEGRA Collaboration], Astron. Astrophys. 393, L37 (2002) arXiv:astro-ph/0207528; F. Aharonian et al. [HEGRA Collaboration], Astron. Astrophys. 431, 197 (2005) arXiv:astro-ph/0501667.

[8] L. A. Anchordoqui, J. F. Beacom, Y. M. Butt, H. Goldberg, S. Palomares-Ruiz, T. J. Weiler and J. Wesolowski, Proc. of the 30th Int. Cosmic Ray Conf., Mérida, México, 3-11 July 2007, Universidad Nacional Autónoma de México, México City, México, 2008, Vol. 2 (OG part 1) 625 arXiv:0706.0517 [astro-ph]].

[9] F. Aharonian [HESS Collaboration], Astron. Astrophys. 467, 1075 (2007) arXiv:astro-ph/0703427.

[10] J. Knödlseder, Astron. Astrophys. 360, 539 (2000). arXiv:astro-ph/0007442.

[11] J. Albert et al. [MAGIC Collaboration], Astrophys. J. 675, L25 (2008) arXiv:0801.2391 [astro-ph]].

[12] W. B. Atwood et al. [LAT Collaboration], Astrophys. J. 697, 1071 (2009) arXiv:0902.1089 [astro-ph.IM]].

[13] A. A. Abdo et al., Astrophys. J. 658, L33 (2007) arXiv:astro-ph/0611691.

[14] A. V. Torres-Dogden, M. Tapia and M. Carroll, Mon. Not. Roy. Astron. S. 249, 1 (1991); P. Massey and A. B. Thompson Astron. J. 101, 4 (1991).

[15] The superposition of the isochrones (calculated using the theoretical evolutionary tracks [16]) on the HertzsprungRussell diagrams, suggests the age of the association is 1-4 Myr [17]. This range reflects the dispersion of the upper main sequence and agrees with the fact that the observed large number of O-type stars implies that the association should be younger than $\sim 5 \mathrm{Myr}$, because in the case of coeval star formation, the number of this type of stars decreases rapidly. In addition, the presence of some Wolf-Rayet stars within Cyg OB2 implies an age larger than $\sim 2 \mathrm{Myr}$, while the non-detection of any supernova remnant [18] points to an association younger than $\sim 4$ Myr.

[16] G. Meynet, A. Maeder, G. Schaeller, D. Schaerer and C. Charbonnel, Astron. Astrophys. Suppl. Ser. 103, 97 (1994).

[17] J. Knödlseder, M. Cerviño, J.-M. Le Duigou, G. Meynet, D. Schaerer and P. von Ballmoos, Astron. Astrophys. 390, 945 (2002) arXiv:astro-ph/0206045.

[18] H. J. Wendker, L. A. Higgs and T. L. Landecker, Astron. Astrophys. 241, 551 (1991).

[19] Y. Butt et al., Astrophys. J. 597, 494 (2003) arXiv:astro-ph/0302342.

[20] D. F. Torres, E. Domingo-Santamaria and G. E. Romero, Astrophys. J. 601, L75 (2004) arXiv:astro-ph/0312128.
[21] L. Yao, E. R. Seaquist, N. Kuno and L. Dunne, Astrophys. J. 588, 771 (2003) [Erratum-ibid. 597, 1271 (2003)] arXiv:astro-ph/0301511.

[22] Y. M. Butt, N. Schneider, T. M. Dame and C. Brunt, Astrophys. J. 676, L123 (2008) arXiv:0802.2964 [astro$\mathrm{ph}]$,

[23] J. Cortina et al. [MAGIC Collaboration], Proc. of the 29th Int. Cosmic Ray Conf. (ICRC 2005), Pune, India, 3-11 Aug 2005, 5 astro-ph/0508275.

[24] J. Holder et al. [VERITAS Collaboration], Astropart. Phys. 25, 391 (2006) arXiv:astro-ph/0604119.

[25] A. M. Lebedev, S. A. Slavatinskii and B. V. Tolkachev, Sov. Phys. JETP 19, 1452 (1963).

[26] S. R. Kelner, F. A. Aharonian and V. V. Bugayov, Phys. Rev. D 74, 034018 (2006) [Erratum-ibid. D 79, 039901 (2009)] arXiv:astro-ph/0606058.

[27] R. S. Fletcher, T. K. Gaisser, P. Lipari and T. Stanev, Phys. Rev. D 50, 5710 (1994).

[28] F. Abe et al. [CDF Collaboration], Phys. Rev. D 41, 2330 (1990).

[29] F. W. Stecker, Phys. Rev. 180, 1264 (1969).

[30] This choice of $n^{\mathrm{HEGRA}}(\epsilon)$ agrees with an estimate in which the densities at the sources are diminished by a common factor $\left\langle 1 / r_{i j}{ }^{2}\right\rangle$, where the latter is simply the average value of the inverse square of the star spacings, taken over the core of the association [ $[\underline{6}$. This is an overly conservative estimate of the fluctuation: if about $10 \%$ of the $\mathrm{O}$ and $\mathrm{B}$ stars are within the $3 \mathrm{pc}$ radius of the HEGRA source, the UV photon population in this region due to emission from these stars alone can be calculated to be $2.5 n^{\star}(\epsilon)$.

[31] V. L. Korotkikh, E. L. Yadrovskii and V. V. Varlamov, Nuclear Physics Institute, Moscow State University, Preprint No. 88-33/54 (1988); I. V.Moskalenko and O. V. Fotina, Sov. J. Nucl. Phys. 49, 1005 (1989) [Yad. Fiz. 49, 1623 (1989)].

[32] A. A. Abdo et al. [Fermi LAT Collaboration], Astrophys. J. Suppl. 183, 46 (2009). arXiv:0902.1340 [astroph.HE]].

[33] E.g., 3EG J2033+4118, R. C. Hartman et al. [EGRET Collaboration], Astrophys. J. Suppl. 123, 79 (1999). A graphic comparison of the EGRET and HEGRA data can be seen in W. Bednarek, Mon. Not. Roy. Astron. Soc. 345, 847 (2003) arXiv:astro-ph/0307216.

[34] S. Funk, O. Reimer, D. F. Torres and J. A. Hinton, Astrophys. J. 679, 1299 (2008) arXiv:0710.1584 [astro-ph]].

[35] A. A. Abdo et al., Science 325, 840 (2009).

[36] F. A. Aharonian et al. [HESS Collaboration], Astron. Astrophys. 442, L25 (2005) arXiv:astro-ph/0510394.

[37] Y. Butt, J. Drake, P. Benaglia, J. Combi, T. Dame, F. Miniati and G. Romero, Astrophys. J. 643, 238 (2006) arXiv:astro-ph/0509191. See also Ref. [19].

[38] L. A. Anchordoqui, H. Goldberg, F. Halzen and T. J. Weiler, Phys. Lett. B 593, 42 (2004) arXiv:astro-ph/0311002.

[39] L. A. Anchordoqui, H. Goldberg, M. C. Gonzalez-Garcia, F. Halzen, D. Hooper, S. Sarkar and T. J. Weiler, Phys. Rev. D 72, 065019 (2005) arXiv:hep-ph/0506168.

[40] A. Reimer, 28th International Cosmic Ray Conference, (Universal Academy Press, Inc., 2005), pp. 2505-2508; E. Domingo-Santamaria and D. F. Torres, Astron. Astrophys. 448, 613 (2006) arXiv:astro-ph/0510769.

[41] G. Hermann, W. Hofmann, T. Schweizer and M. Teshima [CTA Collaboration], arXiv:0709.2048 [astro-ph]. 